\section{Satisfacción usuaria en urgencia oftalmológica: evaluación de la consistencia interna y validez de constructo de un instrumento aplicado en Chile}

\author{
FABIOLA WERLINGER C. ${ }^{1,2,3, a}$, FRANCISCO ANDRIGHETTI C. ${ }^{4}$, \\ NICOLÁS SALAZAR S..$^{1, b}$, IGNACIO CERNA S. ${ }^{1, b}$, \\ MIGUEL CAMPOS G. ${ }^{4, b}$, PATRICIO BUSTAMANTE V.,
}

\section{Evaluation of an instrument assessing the satisfaction of ophthalmological emergency service users in Chile}

Background: Ocular trauma is a distressing condition feared by the general public. Aim: To evaluate the construct validity and internal consistency of an instrument to measure the level of user satisfaction in patients treated at an Ocular Trauma and Ophthalmological Emergency Unit. Material and Methods: A survey to measure user satisfaction was applied to patients consulting at the Ocular Trauma Unit in a public hospital on April 2014. Internal consistency and construct validity from the estimated Cronbach alpha coefficient and factor analysis were assessed. Results: Surveys answered by 138 patients aged $45 \pm 15$ years (93\% men), were analyzed. The reliability obtained for the final questionnaire was 0.88 . The factor analysis yielded four factors responsible for explaining $74.1 \%$ of the total variance, related to "quality of clinical information", "treatment by health care personnel", "lighting conditions, environment and noise" and "waiting time and overall assessment of service". Conclusions: These results allow to consider the instrument as a useful and reliable tool that can be applied to ophthalmological emergency service users in Chile.

(Rev Med Chile 2017; 145: 49-54)

Key words: Emergencies; Ophthalmology; Patient Satisfaction; Quality of Health Care; Validation Studies.

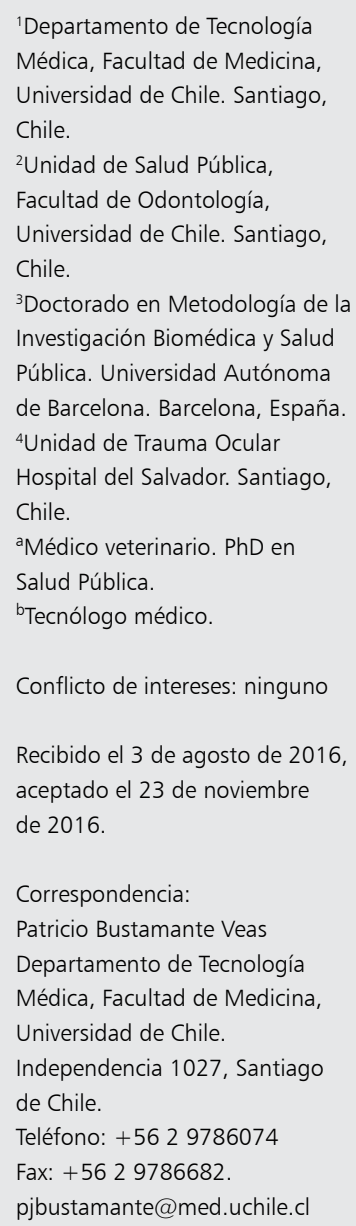

hile enfrenta el desafío de instalar una nueva relación entre las personas y la institucionalidad pública de salud, enfocándose en la percepción de la población usuaria respecto de la calidad de la atención sanitaria ${ }^{1}$. La información brindada por los usuarios y usuarias de los distintos servicios sanitarios, recogida en encuestas especialmente diseñadas para este efecto, se ha vuelto fundamental para realizar una mejor gestión de calidad, orientada al diagnóstico certero de las necesidades y expectativas de la población ${ }^{1,2}$.

Actualmente existe un auge en la realización de estudios que evalúan la satisfacción del usuario con los servicios recibidos, evaluando dimensiones que van desde la infraestructura, hasta la relación del profesional de la salud con los usuarios, siendo éste un indicador de resultado y calidad percibida 
que puede incluso afectar directamente la adherencia al tratamiento ${ }^{3}$.

De esta manera, usuarios satisfechos son proclives a volver al mismo proveedor, recomendarlo a sus familias y mantener relaciones a largo plazo, con una alta vinculación que, desde la perspectiva de la institución de salud, permitirá la implementación de medidas que mejoren la calidad de la atención usuaria y brindar así un mejor servicio ${ }^{3,4}$.

El hecho de medir la calidad percibida a través de la satisfacción usuaria, como dimensión de la calidad en urgencia, ha impulsado cambios en el manejo y dirección de organizaciones sanitarias, que modifican sus enfoques y estrategias hacia una atención centrada en los usuarios que mejoren la accesibilidad, la disponibilidad, la eficiencia, la eficacia y, en definitiva, la calidad en los cuidados de los usuarios ${ }^{5,6}$.

De acuerdo a estos antecedentes, se hace fundamental para evaluar la satisfacción usuaria y la calidad percibida, un instrumento que garantice la eficacia, consistencia y validez en su medición; elementos que en la actualidad han sido trabajados de manera más bien limitada, al menos en el ámbito hispanoamericano ${ }^{7,8}$.

El objetivo de este estudio fue evaluar la validez de constructo y consistencia interna de un instrumento dirigido a medir la satisfacción usuaria de manera consistente, confiable y objetiva, en población chilena atendida en la Unidad de Trauma Ocular y Urgencia Oftalmológica del Hospital del Salvador (UTO) en Santiago de Chile.

\section{Material y Métodos}

Se diseñó un estudio transversal en la UTO, centro de referencia nacional de urgencia oftalmológica para las y los usuarios provenientes de unidades de urgencia de la red de atención de salud de todas las regiones de Chile. Actualmente, la tasa de consulta anual en esta unidad se estima en 33.000 atenciones, más 1.600 cirugías realizadas a usuarios derivados con interconsulta por sospecha de patología ocular traumática y urgencia oftalmológica9 .

Para medir la satisfacción usuaria, dos encuestadores aplicaron un cuestionario a cada paciente al momento de ser dado de alta, durante el período marzo- abril de 2014, previa entrega de un consentimiento informado. La construcción de los contenidos de este instrumento se había realizado en una etapa previa del estudio mediante método Delphi. En esta fase se incluyó un panel de expertos conformado por 6 médicos oftalmólogos y 5 tecnólogos médicos con mención en Oftalmología y Optometría, todos especialistas en urgencia oftalmológica y trauma ocular con al menos 5 años de experiencia profesional.

El cuestionario incluía variables sociodemográficas además del lugar de ocurrencia del trauma, el establecimiento de derivación y si el paciente acudía por primera vez a la unidad. Adicionalmente se incorporó la categorización de la prioridad de la patología del consultante mediante un TRIAGE basado en colores, donde el color amarillo indicaba una lesión ocular urgente, el verde una lesión de riesgo intermedio y el blanco una lesión ocular de menor urgencia (según Urrejola A, Vaillant A., en: "Validación de TRIAGE Oftalmológico en la Unidad de Trauma Ocular del Hospital del Salvador”. Tesis para optar a Título Profesional de Tecnólogo Medico con mención en Oftalmología y Optometría. Escuela de Tecnología Médica. Universidad de Chile. 2010).

Para evaluar la satisfacción se consideraron otras variables, como la valoración del tiempo para ver al médico (correcto/excesivo), si se atendió con respeto al paciente (sí/no), si volvería a venir al servicio (sí/no), si se ayudó a controlar el dolor (sí/no) y si fue informado sobre el tratamiento o controles a seguir al final de la atención (sí/no). En las demás preguntas, el usuario calificaba en un puntaje de 1 a 4 como "muy bueno", "bueno", "malo" y "muy malo" aquellos aspectos relacionados con el trato e información recibidos del personal (administrativo, tecnólogo médico, médico y paramédico) y la valoración de las instalaciones (limpieza, temperatura ambiente/ventilación y luz/condiciones ambientales). Finalmente, se incluía una valoración global del servicio con la misma puntuación para las opciones: "muy satisfactoria", "satisfactoria", "insatisfactoria" o "muy insatisfactoria”.

Se procedió a la evaluación de la consistencia interna a partir de la estimación del coeficiente alfa de Cronbach para cada ítem y la totalidad del cuestionario. La selección de ítems consideró aquellos que presentaron una correlación ítem-total mayor o igual a 0,4 , sin aumentar el estadístico alfa Cronbach al ser eliminado del cuestionario.

Para establecer la validez de constructo del ins- 
trumento, se evaluó la correlación existente entre cada ítem aplicando el coeficiente de correlación de Pearson o Spearman según correspondiera $(\mathrm{p}<0,05)$. Posteriormente, se realizó un análisis factorial con el método de componentes principales para la extracción de factores. Se estableció como punto de corte para su inclusión, valores principales (eigenvalue) mayores o iguales a uno ${ }^{10}$. Para facilitar la lectura de la agrupación de ítems, se realizó la rotación del modelo (método "varimax") seleccionando aquellos que presentaban cargas mayores a $0,4^{10}$. Para evaluar la distribución normal de los datos se aplicó la prueba Shapiro-Wilk. El proceso de análisis fue realizado en el software Stata $11.0^{\circledR}$ (StataCorp, USA).

Todos los procedimientos fueron aprobados por el Comité de Ética del Hospital del Salvador, previo al inicio del estudio.

\section{Resultados}

La muestra final en la que se aplicó el cuestionario quedó constituida por 138 usuarios atendidos en la UTO del Hospital del Salvador con una media de edad de 44,8 $\pm 14,78$ años. Las características del grupo se resumen en la Tabla 1.

\section{Tabla 1. Características de los pacientes consultados, atendidos en la UTO Hospital del Salvador}

\begin{tabular}{|c|c|c|}
\hline Características & $\mathbf{n}$ & $\%$ \\
\hline \multicolumn{3}{|l|}{ Sexo } \\
\hline Mujer & 10 & 7,3 \\
\hline Hombre & 128 & 92,7 \\
\hline \multicolumn{3}{|c|}{ Lugar donde ocurrió el trauma } \\
\hline Lugar de trabajo & 64 & 46,4 \\
\hline Otro lugar & 74 & 53,6 \\
\hline \multicolumn{3}{|l|}{ Previsión } \\
\hline Pública (Fonasa) & 112 & 81,1 \\
\hline Privada (Isapre) & 2 & 1,5 \\
\hline Sin previsión & 24 & 17,4 \\
\hline \multicolumn{3}{|l|}{ Derivación } \\
\hline Hospital público & 70 & 50,7 \\
\hline Atención primaria & 66 & 47,9 \\
\hline Clínica privada & 2 & 1,4 \\
\hline \multicolumn{3}{|l|}{ Vez que asiste a la UTO } \\
\hline Primera vez & 91 & 65,9 \\
\hline Segunda vez o más & 47 & 34,1 \\
\hline \multicolumn{3}{|l|}{ TRIAGE asignado } \\
\hline Blanco & 130 & 94,2 \\
\hline Verde & 5 & 3,6 \\
\hline Amarillo & 3 & 2,2 \\
\hline
\end{tabular}

Tabla 2. Evaluación de la confiabilidad de los ítems seleccionados en el cuestionario de satisfacción

\begin{tabular}{|lcc|}
\hline Ítems & $\begin{array}{c}\text { Correlación } \\
\text { ítem-total }\end{array}$ & $\begin{array}{c}\text { Alfa de } \\
\text { Cronbach }\end{array}$ \\
\hline 1. El tiempo en ser atendido por el médico lo considera correcto o excesivo & 0,192 & 0,895 \\
\hline 2. Cómo calificaría el trato recibido por el personal administrativo & 0,563 & 0,852 \\
\hline 3. Cómo calificaría el trato recibido por el personal paramédico & 0,763 & 0,863 \\
\hline 5. Cómo calificaría el trato recibido por el personal tecnólogo médico & 0,688 & 0,868 \\
\hline 6. Cómo calificaría el trato recibido por el personal médico & 0,754 & 0,864 \\
\hline 7. Cómo considera la información recibida por el personal administrativo & 0,701 & 0,867 \\
\hline 8. Cómo considera la información recibida por el personal paramédico & 0,782 & 0,842 \\
\hline 9. Cómo considera la información recibida por el personal tecnólogo médico & 0,765 & 0,863 \\
\hline 10. Cómo considera la información recibida por el personal médico & 0,760 & 0,864 \\
\hline 11. Su valoración de la limpieza de las instalaciones ha sido & 0,639 & 0,871 \\
\hline 12. Su valoración de la temperatura ambiente de las instalaciones ha sido & 0,667 & 0,869 \\
\hline 13. Su valoración de la luz y condiciones ambientales (ruido) de las instalaciones ha sido & 0,519 & 0,878 \\
\hline 14. Cómo puntuaría globalmente la atención recibida en el servicio de urgencias & 0,433 & 0,883 \\
\hline Alpha de Cronbach & 0,880 \\
\hline
\end{tabular}


Tabla 3. Factores retenidos y varianza asociadas

\begin{tabular}{|llcc|}
\hline Factor & Categoría & Eigen value & Varianza total explicada (\%) \\
\hline Factor 1 & Calidad de la información clínica & 5,75 & $44,3 \%$ \\
\hline Factor 2 & Trato del personal sanitario & 1,43 & $11,0 \%$ \\
\hline Factor 3 & Condiciones de iluminación, ambiente y ruido & 1,26 & $9,7 \%$ \\
Factor 4 & Tiempo de espera y valoración global del servicio & 1,19 & $9,1 \%$ \\
\hline
\end{tabular}

Tabla 4: Estructura de factores retenidos rotados (método varimax)

\begin{tabular}{|c|c|c|c|c|}
\hline & $\begin{array}{c}\text { Factor } \\
1\end{array}$ & $\begin{array}{c}\text { Factor } \\
2\end{array}$ & $\begin{array}{c}\text { Factor } \\
\mathbf{3}\end{array}$ & $\begin{array}{c}\text { Factor } \\
4\end{array}$ \\
\hline $\begin{array}{l}\text { Calidad de la información clínica } \\
\text { Cómo considera la información recibida por el personal administrativo } \\
\text { Cómo considera la información recibida por el personal paramédico } \\
\text { Cómo considera la información recibida por el personal tecnólogo } \\
\text { Cómo considera la información recibida por el personal medico }\end{array}$ & $\begin{array}{l}0,668 \\
0,830 \\
0,865 \\
0,872\end{array}$ & & & \\
\hline $\begin{array}{l}\text { Trato del personal sanitario } \\
\text { Cómo calificaría el trato recibido por el personal administrativo } \\
\text { Cómo calificaría el trato recibido por el personal paramédico } \\
\text { Cómo calificaría el trato recibido por el personal tecnólogo } \\
\text { Cómo calificaría el trato recibido por el personal medico }\end{array}$ & & $\begin{array}{l}0,711 \\
0,812 \\
0,851 \\
0,732\end{array}$ & & \\
\hline $\begin{array}{l}\text { Condiciones de iluminación, ambiente y ruido } \\
\text { Su valoración de la limpieza de las instalaciones ha sido } \\
\text { Su valoración de la temperatura ambiente de las instalaciones ha sido } \\
\text { Su valoración de la luz y condiciones ambientales (ruido) de las instalaciones ha sido }\end{array}$ & & & $\begin{array}{l}0,704 \\
0,778 \\
0,775\end{array}$ & \\
\hline $\begin{array}{l}\text { Tiempo de espera y valoración global del servicio } \\
\text { El tiempo en ser atendido por el médico lo considera correcto o excesivo } \\
\text { Cómo puntuaría globalmente la atención recibida en el servicio de urgencias }\end{array}$ & & & & $\begin{array}{l}0,788 \\
0,558\end{array}$ \\
\hline
\end{tabular}

Al explorar la correlación de cada ítem con la escala completa (item-test correlation), esta fue baja (menor a 0,4 ) para las preguntas dirigidas al tiempo de atención, control del dolor, información final entregada y si volvería a venir al mismo servicio, por lo que se retiraron de la selección, exceptuando el tiempo de espera. Dado que, además, todos los encuestados manifestaron haber sido atendidos con respeto, se eliminó el ítem referido a esta condición, por no presentar variabilidad. En la Tabla 2 se presentan los resultados de la evaluación de la fiabilidad del cuestionario con las variables seleccionadas, el que alcanzó un coeficiente alfa de Cronbach de 0,880.

Al aplicar el análisis factorial (Tabla 3) se pudo establecer la predominancia de 4 factores responsables de explicar el $74,1 \%$ de la varianza total (índice Kaiser-Meyer-Olkinde 0,83). De acuerdo a su estructura, estos fueron denominados como "calidad de la información clínica", "trato del personal sanitario", "condiciones de iluminación, ambiente y ruido" y "tiempo de espera y valoración global del servicio” (Tabla 4).

\section{Discusión}

Nuestros resultados se condicen con lo descrito por la literatura respecto del tipo de población afectada por trauma ocular, la que indica una mayor probabilidad de ocurrencia en los hombres, asociada al tipo de trabajo desarrollado con mayor contacto físico y comportamiento impulsivo ${ }^{10-12}$. Es interesante observar que, aunque en nuestra muestra la edad media de los usuarios corresponde a una edad laboral activa (44,8 años), esta fue 
mayor a la estimada en otras poblaciones donde se describen edades en torno a los 30 años ${ }^{11-14}$.

En relación a la confiabilidad del instrumento, se decidió retirar aquellas variables que se encontraron por debajo del punto de corte establecido a priori y que ha sido descrito como cuestionable por la literatura relacionada a escalas psicométricas $^{10,15,16}$.

Esto consideró excluir las preguntas correspondientes a la información entregada al final de la atención y si el usuario volvería a venir al mismo servicio, al ser entendidas como condiciones prescindibles en este cuestionario y con una escasa relación con la satisfacción usuaria en este grupo de estudio.

Conjuntamente, si bien el control del dolor ha sido reportado como una condición relevante para la satisfacción en otras experiencias en el ámbito hospitalario ${ }^{17,18}$, en nuestro caso, la baja correlación observada con el resto de las preguntas del cuestionario fue atribuida a las características de las lesiones predominantes en los consultantes en esta unidad de urgencia, donde principalmente se recepcionan lesiones oculares de carácter leve, consistente con el tipo de TRIAGE asignado (blanco y verde), razón por la cual también se decidió eliminar.

La estimación de la confiabilidad sin estas tres variables arrojó finalmente un coeficiente mayor $(0,880)$, que fue considerado como excelente ${ }^{19}$, en términos de confiabilidad para el cuestionario resultante.

A diferencia de las condiciones descritas, la decisión respecto de mantener la pregunta sobre el tiempo de espera, se basó en la relevancia reportada en otras encuestas de satisfacción usuaria aplicadas en poblaciones similares a la nuestra (latinoamericana e hispana $)^{15,20}$, donde tal condición fue considerada como relevante en la calidad de la atención de salud y como indicador del acceso de los usuarios a los servicios sanitarios ${ }^{6}$.

Posteriormente, la evaluación de la validez de constructo según el análisis del factor permitió agrupar las preguntas seleccionadas de este cuestionario en cuatro dimensiones entendidas como las responsables de predecir la satisfacción usuaria en esta unidad de trauma ocular (Tabla 3).

El primer factor, denominado "calidad de información clínica”, fue el elemento más relevante para este grupo de estudio e incluyó aquellos aspectos relacionados con la información entregada directamente al paciente, englobando al personal clínico y administrativo del servicio (Tabla 4). Estos resultados, al igual que otros reportes ${ }^{15,17,21,22}$, indicarían que mientras la calidad de la información otorgada a los usuarios sea mayor, la valoración global de la atención brindada también será mejor. Tal análisis releva la importancia de entregar una información clínica adecuada, desafiando a los servicios de salud a establecer medidas y protocolos para este proceso en sus profesionales.

El "trato del personal sanitario" y las "condiciones de luz, ambiente y ruido" fueron también relevados como elementos importantes para la satisfacción, tal y como se explica en otros estudios con estadías más largas en los servicios sanitarios como es la hospitalización ${ }^{15,17,21,22}$.

Por último, el cuarto factor reunió las variables de "tiempo de espera y valoración global del servicio" como experiencia compleja y multidimensional. En este sentido, corrobora la relación establecida entre el tiempo de espera percibido por el usuario para recibir atención médica y la satisfacción usuaria, teniendo directa relación con la valoración global del servicio ${ }^{20,21}$.

Según estos resultados, es posible afirmar que el cuestionario elaborado para medir satisfacción usuaria en la Unidad de Trauma Ocular del Hospital del Salvador en Santiago de Chile es útil y confiable, reuniendo aspectos relacionados al trato y entrega de información del personal, las condiciones del entorno y el tiempo de atención como los elementos más relevantes para los usuarios.

Esta es la primera encuesta de satisfacción usuaria validada en urgencia oftalmológica en Chile lo que, sin duda, es un primer paso en la generación de herramientas que permitan desarrollar un adecuado proceso de gestión y mejoramiento continuo de la calidad de un servicio único, como es la Unidad de Trauma Ocular del Hospital del Salvador, centro de derivación nacional. Visibilizar este tipo de información puede contribuir al cumplimiento de los objetivos sanitarios de la Estrategia Nacional de Salud 2011-2020 en Chile.

Se sugiere su aplicación en diversas oportunidades a futuro, con el fin de evaluar constantemente la satisfacción de los usuarios y usuarias a lo largo del tiempo y de ese modo contribuir al mejoramiento de la calidad en salud. Es posible además considerar la extrapolación de esta encuesta a otros servicios de urgencias interesados en evaluar la satisfacción usuaria. 


\section{Referencias}

1. Gobierno de Chile. Estrategia Nacional de Salud 2011-2020 [monografía en internet] 2013. Disponible en: http://www.salud-e.cl/biblioteca/estrategia-sanitaria-chilena/objetivos-sanitarios-2011-2020/ [consultado el 12 de noviembre de 2015].

2. Ministerio de Salud (MINSAL) Gobierno de Chile (2009). Medición Nacional de Satisfacción Usuaria en la red Pública de Salud de Chile. Departamento de Participación Social y Trato al Usuario, Subsecretaría de Redes Asistenciales, MINSAL.

3. Mira J, Aranaz J. La Satisfacción del paciente como una medida del resultado de la atención sanitaria. Med Clin (Barc) 2000; 114 (3): 26-33.

4. Heidegger T, Detlef S, Nuebling M. Patient satisfaction with anaesthesia care: What is patient satisfaction, how should it be measured, and what is the evidence for assuring high patient satisfaction? Best Prac Res Clin Anesthesiol 2006; 20 (2): 331-46.

5. Bustamante V, Alvarado O, Romero A. Evaluación de la satisfacción del paciente con la atención recibida en un servicio de urgencias hospitalario y sus factores asociados. Rev Emergencias 2013; (3): 171-6.

6. Brown LD, Franco L, Rafeh N, Hatzell T. Garantía de calidad de la atención de salud en los países en desarrollo. Segunda edición. [Internet]. Disponible en: http://pdf. usaid.gov/pdf_docs/Pnach089.pdf [consultado el 12 de noviembre de 2015].

7. Carvajal A, Centeno C, Watson R, Martínez M, Rubiales A. How is an instrument for measuring health to be validated? An Sist Sanit Navar 2011; 34 (1): 63-72.

8. Bucchi C, Sepúlveda C, Monsalves M, Bustos L. Descripción de la Satisfacción Usuaria de Pacientes que Reciben Atención de Urgencia Dental en Cinco Establecimientos de Atención Primaria de Salud. Int J Odontostomat 2012; 6 (3): 275-80.

9. Hospital del Salvador, SSMO Ministerio de Salud, UNIDAD TRAUMA OCULAR (UTO). Disponible en: http://www.hsalvador.cl/unidad-trauma-ocular-uto/ [Citado el 19 de septiembre de 2013].

10. Acock A. Measurement, reliability, and validity. En: $A$ gentle introduction to Stata. Tercera edición. Texas, USA: Stata Press 2012; 325-56.
11. May DR, Kuhn FP, Morris RE, Whiterspoon CD, Danis RP, Matthews GP, et al. The epidemiology of serious eye injuries, from the United States Eye Injury Registry. Graefe's Arch Clin Exp Ophthalmol 2000; 238 (2): 1537.

12. Klopfer J, Tielsch J, Vitale S, See L, Canner J. Ocular Trauma in the United States, Eye Injuries Resulting in Hospitalization, 1984 Through 1987. Arch. Ophtalmol 1992; 110 (6): 838- 42.

13. Sánchez R, Piccevic D, León A, Ojeda M. Trauma Ocular. Cuad Cir 2008; 22: 91-7.

14. Ministerio de Salud. Guía Clínica Trauma Ocular Grave. Santiago: Minsal, 2007.

15. Calixto-Olalde M, Okino N, Hayashida M, Costa I, Trevizan M, Godoy S. Escala SERVQUAL: validación en población mexicana. Texto contexto-enferm 2011; 20: 526-33.

16. Matkar A. Cronbach's Alpha Reliability Co-efficient for standard of customer services in Maharashtra State Co-operative Bank IUP. Journal of Bank Management 2012; 11(3): 89.

17. Castillo L, Dougnac A, Vicente I, Muñoz V, Rojas V. Los predictores de satisfacción de pacientes en un centro Hospitalario Universitario. Rev Med Chile 2007; 135 (6): 696-701.

18. Andrés M, García- Castillo L, Rubini S, Juárez R, Scaf E, Fernández M, et al. Evaluación del efecto de la información en la satisfacción de los pacientes atendidos en los servicios de urgencias hospitalarios. Rev Calidad Asistencial. 2007; 22 (4): 161-7.

19. Streiner D. Starting at the beginning: an introduction to coefficient alpha and internal consistency. J Pers Assess 2003; 80: 99-103.

20. Carbonell M, Girbés J, Calduch J. Determinantes del tiempo de espera en urgencias hospitalarias y su relación con la satisfacción del usuario. Rev. Emergencias 2006; 18: 30-3.

21. Parra P, Bermejo R, Más A, Hidalgo M, Gomis R, Calle $\mathrm{J}$. Factores relacionados con la satisfacción del paciente en los servicios de urgencias hospitalarios. Gac Sanit 2012; 26 (2): 159-65.

22. Mira J, et al. Causas de satisfacción y de insatisfacción de los pacientes en hospitales y atención Primaria. Rev Calidad Asistencial 2002; 17 (5): 273-83. 